\title{
The prevalence and treatment outcomes of antineuronal antibody-positive patients admitted with first episode of psychosis
}

James G. Scott, David Gillis, Alex E. Ryan, Hethal Hargovan, Nagaraj Gundarpi, Gemma McKeon, Sean Hatherill, Martin P. Newman, Peter Parry, Kerri Prain, Sue Patterson, Richard C. W. Wong, Robert J. Wilson and Stefan Blum

\section{Background}

Antineuronal antibodies are associated with psychosis, although their clinical significance in first episode of psychosis (FEP) is undetermined.

\section{Aims \\ To examine all patients admitted for treatment of FEP for anti- neuronal antibodies and describe clinical presentations and treatment outcomes in those who were antibody positive.}

\section{Method}

Individuals admitted for FEP to six mental health units in Queensland, Australia, were prospectively tested for serum antineuronal antibodies. Antibody-positive patients were referred for neurological and immunological assessment and therapy.

\section{Results}

Of 113 consenting participants, six had antineuronal antibodies (anti- $N$-methyl-D-aspartate receptor antibodies $[n=4]$, voltagegated potassium channel antibodies $[n=1]$ and antibodies against uncharacterised antigen $[n=1]$ ). Five received immunotherapy, which prompted resolution of psychosis in four.

\section{Conclusions}

A small subgroup of patients admitted to hospital with FEP have antineuronal antibodies detectable in serum and are responsive to immunotherapy. Early diagnosis and treatment is critical to optimise recovery.

\section{Declaration of interest}

None.

\section{Copyright and usage}

(c) The Royal College of Psychiatrists 2018. This is an Open Access article, distributed under the terms of the Creative Commons Attribution-NonCommercial-NoDerivatives licence (http://creativecommons.org/licenses/by-nc-nd/4.0/), which permits noncommercial re-use, distribution, and reproduction in any medium, provided the original work is unaltered and is properly cited. The written permission of Cambridge University Press must be obtained for commercial re-use or in order to create a derivative work.
For some years, blockade of $N$-methyl-D-aspartate receptors (NMDAR) has been associated with the pathogenesis of psychosis. ${ }^{1}$ Inhibiting antibodies to NMDAR (anti-NMDAR antibodies) in humans were first described in a group of females with teratoma and severe limbic encephalitis with a psychiatric prodrome. ${ }^{2}$ AntiNMDAR antibodies have subsequently been found in a broader population of patients with encephalitis, including males and children with and without demonstrable malignancy and with a variety of clinical features, including psychosis as a sole presenting feature. Many of these people present to mental health services initially, before the autoimmune nature of their illness becomes manifest, with numerous case reports and small case series identifying anti-NMDAR encephalitis in those presenting with an initial diagnosis of psychosis. ${ }^{3-10}$ Although early detection and initiation of immunotherapy is important to improve their long-term outcome, ${ }^{11,12}$ presentation to mental health services potentially delays diagnosis and appropriate treatment. In contrast, detection of classical anti-NMDAR encephalitis is rarely described in established schizophrenia and related psychotic disorders. ${ }^{13-15}$ However, IgA and IgM antibodies against NMDAR, atypically found in anti-NMDAR encephalitis, have been detected in people diagnosed with schizophrenia. ${ }^{15,16}$ Less commonly, cases have been reported of individuals with voltage-gated potassium channel antibodies $\left(\right.$ anti-VGKC ${ }^{17}$ ). Despite the above reports, the clinical significance of detecting anti-NMDA receptor and VGKC antibodies in patients presenting with first episode of psychosis (FEP) remains unclear, and it is uncertain if providing immunomodulatory treatment to patients presenting with psychosis and antineuronal antibodies improves outcomes. Lennox and colleagues reported nine percent of patients with FEP had serum antineuronal antibodies. ${ }^{18}$ None of these were diagnosed with encephalitis and the presence of antibodies was not associated with increased need for care over 6 months as defined by admissions, out-patient contacts or crisis service interventions. Immunological interventions were not utilised. We performed a prospective study of all people presenting to three inpatient psychiatry services in southeast Queensland, Australia, with FEP. The aim was to prospectively determine the prevalence of serum antineuronal antibodies, including anti-NMDAR antibodies, in this clinical population and describe antibody-positive patient response to immunotherapy.

\section{Method}

\section{Study sites}

The study was undertaken in six acute psychiatry units located at three hospitals in Brisbane, Australia (The Royal Brisbane and Women's Hospital, The Logan Hospital Adolescent Unit and the Lady Cilento Children's Hospital). Recruitment commenced in July 2013 and participants were recruited from two 26 bed general adult psychiatry units, one 26 bed unit specialising in the care for female patients aged 18-25 years, two 12 bed adolescent units (ages 14-17 years) and one 12 bed acute psychiatry unit for children up to and including 13 years. Recruitment at all sites ended in May 2015.

\section{Participants}

Those aged between 12 and 50 years admitted to one of the three hospitals for the first time for management of their FEP were 
approached to participate in the study. Patients were included in the study if they were definitively suffering from a psychotic disorder (including substance-induced psychotic disorder) as diagnosed by a multidisciplinary team lead by a consultant psychiatrist for which no overt medical condition could be identified. Those who had capacity consented to a blood test for antineuronal antibodies and the recording of clinical and demographic information for the purposes of the study as early as possible after admission. Where the individual lacked capacity, consent was obtained from an 'alternative decision maker', who was usually a family member or partner of the participant. When the participant recovered, they were again approached to explain the study and obtain informed consent. If they declined to participate, their results were not included. Ethics approval was obtained to collect basic demographic (age and gender) and clinical information (diagnosis, duration of untreated psychosis and recruitment site) of patients who refused, so as to compare those who did and did not participate. The study was approved by the Metro South Hospital and Health Service Human Research Ethics Committee (approval number HREC/12/QPAH/598).

\section{Detection of antineuronal antibodies and assessment for autoimmune encephalitis}

All samples were analysed in the Autoimmune Section of Pathology Queensland, which is a referral diagnostic laboratory, offering specialist neuroimmunology testing for the east coast of Australia. All consenting participants had their plasma tested for intracellular antineuronal antibodies (including purkinje cell cytoplasmic antibody type 1 (PCA-1)/anti-Yo, PCA-2, type 1 antineuronal nuclear antibody (ANNA-1)/anti-Hu, ANNA-2/anti-Ri and anti-Ma) by indirect immunofluorescence on a composite slide of primate cerebellum/cerebrum and murine gastric tissues (Inova Diagnostics, USA). Anti-NMDAR IgG antibodies were detected in diluted serum (1:10) as per manufacturer's instructions by a commercial assay containing four biochips of primate hippocampus, primate cerebellum, NMDAR-transfected human embryonic kidney cells 293 (HEK293) and nontransfected control HEK293 cells (Euroimmun, Germany). At Pathology Queensland, antiNMDAR antibody titres are not routinely performed as the commercial NMDAR NR1 subunit-transfected cell lines used by all Australian diagnostic laboratories are not readily amenable for end-point titration. ${ }^{19}$ Low positive results for NMDAR antibodies are detected by weak immunofluorescence staining. Anti-VGKC were detected by a commercial radioimmunoassay (RSR, UK). This quantitative assay utilizes detergent solubilized VGKCs extracted from rabbit brain tissue and complexed with 125I-labelled $\alpha$-dendrotoxin. All positive sera on the radioimmunoassay were then tested by indirect immunofluorescence on biochips of leucine-rich glioma inactivated 1 (LG1) and contactin-associated protein 2 (CASPR2)-transfected HEK293 cells (Euroimmun). Anti-glutamic acid decarboxylase (GAD) antibodies were detected by a commercial quantitative immunoassay (RSR). The assay utilizes GAD65 coated onto the wells of an enzyme-linked immunosorbent assay plate. Sensitivity is increased by a second step that includes the addition of GAD-biotin and a streptavidin detection step.

Where participants were found to be positive for any of the above antineuronal antibodies, consultation was sought from neurologists and immunologists with extensive experience in the treatment of patients with autoimmune encephalitis. Participants were transferred to medical units, where a medical history was obtained, and appropriate investigations were undertaken to confirm or exclude the diagnosis of encephalitis, investigate for a potential paraneoplastic cause and initiate immunotherapy. After diagnosis, antibody-positive patients were followed up by both neurological and psychiatry services.

\section{Results}

\section{Comparison of those who participated and those who refused}

During the period of data collection, 154 patients were admitted to the three hospitals for treatment of their FEP and 113 (73.3\%) consented to the study. Those participating in the study consisted of $66(58.4 \%)$ males, with a mean age of 26.2 years (s.d. 9.2; range 13-50). The median duration of untreated psychosis was 30 days (range 1-2340), with 57 (50.4\%) participants having a duration of untreated psychosis estimated by clinicians to be less than 30 days. The most common diagnoses were schizophrenia, substance-induced psychotic disorder and schizophreniform disorder, which accounted for greater than $50 \%$ of all patients (Table 1). Of those who refused $(n=41), 29(70.7 \%)$ were male, with a mean age of 28.5 years (s.d. 9.6) and a median duration of untreated psychosis of 60 days. A comparison of those included with those who refused to participate revealed no significant differences in age and gender, although nonparticipants had a longer median duration of untreated psychosis.

\section{Description of antineuronal antibody-positive patients}

Of the 113 participants, six tested positive for antineuronal antibodies in serum (Table 2). Of these, four (patients 1-4) had antiNMDAR antibodies, one (patient 5) had antibodies to VGKC with a titre of $203 \mathrm{pMol} / \mathrm{L}$ (by radioimmunoassay alone, with negative anti-LGI1 and anti-CASPR2 on the transfected HEK293 cells) and a final patient (patient 6) had antineuronal antibodies of uncertain clinical relevance to an uncharacterised antigen. Testing of sera from patient 6 showed weak immunofluorescent, fine-speckled staining throughout the cerebellar molecular and granular layers. There was non-specific Purkinje cell staining but no staining of white matter or hippocampal tissue. Other testing revealed the patient was negative for GAD, NMDA, LGI1, CASPR2, AMPA and GABA.

Five of the six patients (patients 1-5) lacked capacity to provide informed consent at time of serum collection and an alternative decision maker was needed to initially enrol these patients into the study as per the protocol. Informed consent was obtained when the patients had later recovered.

All six participants initially presented with acute onset of psychosis. The delay in diagnosis and treatment of patient 3 was because of initial attempts at community management. The progressive escalation in symptoms eventually necessitated admission to hospital despite out-patient psychiatric care. Patients 1-4 had NMDAR antibodies detected within 1 week of commencing psychotropic medication, an inadequate length of treatment to determine

Table 1 Initial psychiatric diagnoses of the 113 participants with first episode of psychosis

$\begin{array}{lc}\text { Diagnosis } & N(\%) \\ \text { Schizophrenia } & 21(18.6) \\ \text { Substance-induced psychotic disorder } & 19(16.8) \\ \text { Schizophreniform psychosis } & 19(16.8) \\ \text { Brief psychotic disorder } & 16(14.2) \\ \text { Bipolar affective disorder } & 15(13.2) \\ \text { Psychotic disorder NOS } & 14(12.4) \\ \text { Other psychotic disorders } & 9(8.0)\end{array}$




\begin{tabular}{|c|c|c|c|c|c|c|c|c|}
\hline $\begin{array}{l}\text { Participant } \\
\text { No. Age/ } \\
\text { Gender }\end{array}$ & Initial Diagnosis ICD-10 $0^{31}$ & $\begin{array}{l}\text { Duration of } \\
\text { Untreated } \\
\text { Psychosis } \\
\text { (Days) }\end{array}$ & Symptoms & Antibody & Seizure & CSF & Initial EEG & MRI \\
\hline 1. $28, F$ & $\begin{array}{l}\text { Substance-induced psychosis } \\
\text { (cannabis) }\end{array}$ & 7 & $\begin{array}{l}\text { Acute confusion, headaches, hallucinations, } \\
\text { agitation, catatonia, encephalopathy with } \\
\text { reduction in level of consciousness } 8 \text { days } \\
\text { after psychosis onset }\end{array}$ & NMDAR & Yes & WCC 50, Prot 360, NMDAR+ & Normal & Normal \\
\hline 2. $16, \mathrm{~F}$ & $\begin{array}{l}\text { Acute and transient psychotic } \\
\text { disorder }\end{array}$ & 5 & $\begin{array}{l}\text { Agitation, confusion, seizures, } \\
\text { encephalopathy with seizures } 9 \text { days after } \\
\text { onset of first symptoms }\end{array}$ & NMDAR & Yes & $\begin{array}{l}\text { WCC 15, Prot 370, OCB+, } \\
\text { NMDAR+ }\end{array}$ & $\begin{array}{l}\text { Fast background, right } \\
\text { temporal slow }\end{array}$ & Normal \\
\hline 3. $13, \mathrm{M}$ & Schizophreniform disorder & 70 & $\begin{array}{l}\text { Irritable, confusion, labile mood, } \\
\text { hallucinations }\end{array}$ & NMDAR & No & $\begin{array}{l}\text { WCC 1, Prot 160, OCB+, NMDA } \\
\text { low+ }\end{array}$ & ND & Normal \\
\hline 4. $33, \mathrm{M}$ & Bipolar affective disorder & 2 & $\begin{array}{l}\text { Suicidal thoughts, delusional thoughts, } \\
\text { hallucinations, depressed mood }\end{array}$ & NMDAR & No & $\begin{array}{l}\text { WCC 35, Prot 450, OCB-, } \\
\text { NMDA- }\end{array}$ & Normal & Normal \\
\hline 5. $16, \mathrm{M}$ & First episode of psychosis & 2 & Bizarre behaviour, thought disorder & VGKC & No & WCC 2, Prot 340, OCB-, NMDA- & $\begin{array}{l}\text { Diffuse slowing of } \\
\text { background }\end{array}$ & Normal \\
\hline 6. $23, \mathrm{M}$ & First episode of psychosis & 7 & Mania, psychosis & Unknown & No & ND & Normal & $\begin{array}{l}\text { ND, Head computed } \\
\text { tomography } \\
\text { scan normal }\end{array}$ \\
\hline
\end{tabular}

\section{Table 3 Overview of antipsychotic and immunotherapy and treatment response}

Patient No.

Age/Gender

1. $28, \mathrm{~F}$

Initial Psychotropic Therapy and Response

Olanzapine and diazepam for 6 days. No improvement with

medications. Some sedation with fluctuation in mental state

Time of Initiation of Immunotherapy

Immunotherapy commenced 6 days after admission for psychosis. Teratoma removal, IVMP, IVIg, RTX.

2. 16, F Olanzapine and diazepam for 4 days. Some reduction in agitation with Immunotherapy commenced 4 days after admission for psychosis. psychotropic medication

3. 13, M Olanzapine for 7 days with no improvement

Teratoma removal, IVMP, IVIg, RTX

Immunotherapy commenced 7 days after admission.

IVMP, IVIg

4. 33, M Initially risperidone and mirtazapine. Akathisia experienced, and Immunotherapy commenced 6 days after admission to hospital.

risperidone ceased. Commenced on quetiapine. No response after IVMP, IVIg, AZA

risperido

Immunotherapy commenced 22 days after admission to hospital. IVIg, IVMP

No immunotherapy
Duration of Follow-Up and Treatment Response

3 years and 9 months: no psychosis. Some symptoms of depression and anxiety. Working full time

2 years and 9 months: no psychosis. Some social difficulties following illness. Attending university

2 years and 6 months: no psychosis. Sleep problems and fluctuating mood. Unemployed

1 year and 6 months: no psychosis. Persistent symptoms of depression and anxiety. Working full time

2 year and 6 months: good response to IVMP. Relapsing course Remains on olanzapine. Attending school full time 1 month: remission from psychosis

6. 23, M Risperidone NO 
response. Patient 5 received risperidone ( $2 \mathrm{mg}$ each night, for 22 days) before immunotherapy was commenced. There was no clinical improvement with this antipsychotic (Table 3).

Two participants (patients 1 and 2) developed a diffuse encephalopathy with seizures consistent with classical limbic encephalitis after the testing of serum for antibodies. Three participants (patients 3-5) had isolated psychiatric syndromes without other features of limbic encephalitis, such as seizures or other neurological signs. These patients may have potentially remained in psychiatric services with undiagnosed autoimmune illnesses. One participant (patient 6) was seen on several occasions in the month after discharge having been treated with risperidone. During this time, there were no mood or psychosis symptoms and he was subsequently lost to follow-up.

Two of four anti-NMDAR antibody-positive patients (patients 1 and 2) had an ovarian teratoma detected and removed; these patients quickly deteriorated clinically in the initial stages of their illness. Anti-NMDAR antibodies as well as oligoclonal bands were detected in the cerebrospinal fluid (CSF) of one participant (patient 3), who responded well to immunotherapy. Another participant (patient 4) had no detectable anti-NMDAR antibodies in CSF, but an increased CSF white cell count was identified. One antibody-positive participant (patient 2) required intubation and therapy in an intensive care setting. Three participants (patients 1, 3 and 4) were treated on a neurology ward for the duration of their acute illness. For the patient who tested positive for VGKC antibodies (patient 5), presence of antibodies to VGKCs was confirmed by repeat testing in a quaternary laboratory (Oxford Neuroimmunology Laboratory, UK). He subsequently had relapsing presentations of psychosis with a good response to immunotherapy utilizing intravenous methylprednisolone and intravenous immunoglobulin.

At last follow-up, participants 1 and 4 had ongoing symptoms of depression and anxiety but were functioning well, engaged in fulltime employment. Participant 2 had some difficulties socially after her illness, although this had improved over time and at last follow-up she was successfully attending tertiary education. Participant 5 had experienced a brief relapse of psychosis when antipsychotic medication had been withdrawn. Participant 3 had no further psychosis but continued to experience sleep disturbance and some mood instability (Table 3 ).

In one participant (patient 6), a previously uncharacterised staining pattern was detected by indirect immunofluorescence on primate cerebellum. This person left hospital soon after recruitment and could not be followed up; the medical work-up was incomplete and no immunotherapy was instituted.

\section{Discussion}

This real-world clinical study prospectively assessed the prevalence of antineuronal antibodies in patients admitted to hospital with FEP and reports their response to immunomodulatory therapy. We recruited 113 people admitted to hospital with FEP in Queensland, Australia, and detected the presence of antineuronal antibodies in six individuals (5.3\%), including four (3.5\%) participants with antiNMDAR antibodies, one $(0.8 \%)$ with anti-VGKC antibodies and one $(0.8 \%)$ with a previously uncharacterised staining pattern on primate cerebellum with indirect immunofluorescence.

Clinical and paraclinical findings support the notion that a central nervous system autoimmune process was present in five of these six participants (patients 1-5). Importantly, neuroimaging with brain magnetic resonance imaging and electroencephalogram did not render abnormal results in any of the antibody-positive patients. Two participants (patients 1 and 2) had a paraneoplastic process in the context of an ovarian teratoma. In patient 3 , antiNMDAR antibodies were detected in CSF, together with oligoclonal bands. In patient 4, anti-NMDAR antibodies were not detected in CSF; however, a lymphocytic pleocytosis was indicative of an inflammatory process. One participant (patient 5) presented with a relapsing-remitting course in the presence of VGKC positivity in the absence of antibodies to LGI1 and CASPR2. Recent consensus suggests this is unlikely to be a clinically significant autoimmune process; ${ }^{20,21}$ however, this participant did respond well to intravenous immunoglobulins and methylprednisolone after having no response to 22 days of low-dose risperidone. Immunotherapy was not commenced in patient 6 as the participant had recovered by the time the serum antibodies were detected. Participants 1-5 received immunotherapy, using different regimens, leading to resolution of psychosis in four patients (Table 3).

These favourable clinical outcomes in patients who presented with FEP with such high acuity as to require inpatient care were similar to a previously reported case series of nine patients with psychosis who received immunotherapy. ${ }^{22}$ Of these nine, six had positive responses. The three nonresponders had long histories ( $>3$ years) of treatment refractory illness. The excellent clinical responses seen in our case series may be a result of the very early provision of immunotherapy.

\section{Relationship of antineuronal antibodies to the pathogenesis of chronic psychotic disorders}

The high prevalence of antineuronal antibodies in FEP contrasts with the low prevalence in established schizophrenia. ${ }^{13,16,23,24}$ However, other studies have reported similar rates of psychosis for which an autoimmune aetiology has been postulated. Steiner and colleagues examined the plasma collected within $24 \mathrm{~h}$ of admission of 121 patients diagnosed with schizophrenia for antibodies to the NR1a or NR1a/NR2b subunits of the NMDA receptors. ${ }^{16}$ In those patients in whom antibodies were detected, CSF was also obtained for testing. In four people, antibodies to the NMDA receptors were identified. Of these, two who were initially diagnosed as having catatonic or disorganised schizophrenia had high titres of IgG NR1a antibodies in both sera and CSF, and were subsequently reclassified as having anti-NMDAR encephalitis.

In a series of 96 women with postpartum psychosis whose plasma was retrospectively tested for antineuronal antibodies, Bergink and colleagues reported two (2\%) had anti-NMDAR encephalitis as diagnosed by the presence of NMDAR antibodies to the NR1a subunit detected with a combination of immunohistochemistry, immunocytochemistry and cell-based assay. ${ }^{10}$ Both patients were sensitive to extra pyramidal side-effects but otherwise did not display clinical features that differentiated them from other women with postpartum psychosis. Both achieved remission without immunotherapy and as a result, CSF was not obtained for testing. In addition, two other patients had antineuronal antibodies to unidentified antigens, suggesting that other autoimmune encephalitides may also be present in a small percentage of patients with postpartum psychosis.

In a large sample $(n=228)$ of patients with FEP, Lennox and colleagues reported that 20 (9\%) had antineuronal antibodies, including 11 (5\%) with VGKC antibodies and 7 (3\%) with NMDAR antibodies. ${ }^{18}$ No patients were diagnosed with encephalitis or developed neurological symptoms as assessed by their treating psychiatrist. Participants in this study were recruited from community and hospital services and the participation rate is unknown. It is possible the most severely ill patients were unable to consent to be included in this study. In our study, the majority of antibody positive patients (patients 1-5) were too unwell to initially consent, requiring an alternative decision maker to enable 
participation. Without this sampling method, those with encephalitis may not have been able to participate.

Together, these studies suggest that a small percentage of people presenting to hospital with acute psychosis have an autoimmune illness best detected by testing sera or CSF for neuronal antibodies. Furthermore, it is possible that an initial, untreated insult presenting phenotypically as acute psychosis could lead to long-term changes in brain function phenotypically consistent with chronic schizophrenia, whilst the initial inflammatory cause resolves spontaneously. There are several well-established, postinfectious immunemediated diseases in which spontaneous normalization of the immune response occurs, with or without detectable autoantibodies, such as in Guillain-Barré syndrome or poststreptococcal chorea. Indeed, although detected and treated cases of antiNMDAR encephalitis do well in $80 \%$ of patients, neurocognitive deficits can persist long after the initial insult. ${ }^{25,26}$

\section{Strengths and limitations}

This study accounted for all patients with a FEP requiring admission to three acute inpatient psychiatric services and engaged a sampling strategy that ensured that the most unwell patients would be included in the study, including accounting for those who refused to participate. It describes the clinical course of the six participants who screened positive for antineuronal antibodies. However, like all research, this clinical study had a number of limitations. There was no healthy control group with whom to compare the prevalence of antineuronal antibodies. However, the current literature suggests that these antibodies are rarely found in nonclinical samples. ${ }^{27,28}$

Another limitation was that the prevalence of autoimmune encephalitis may have been underestimated as antineuronal antibodies, in particular anti-NMDAR antibodies, were not tested in CSF of all recruited participants, but only in those who had antiNMDAR and anti-VGKC antibodies detected in the serum. It has recently even been proposed that in patients with psychosis as the only or predominant clinical manifestation of anti-NMDAR encephalitis, the titre of anti-NMDAR antibodies might be too low to be detectable in the serum, and can only be detected in CSF. ${ }^{29}$ However, when our methodology was designed, it was decided that a requirement to routinely perform a lumbar puncture for CSF on patients with FEP would have rendered the study unfeasible because of patient refusal. We do recommend testing for antineuronal antibodies in the CSF of people with FEP who have symptoms highly suggestive of an autoimmune encephalitis (acute onset of illness, severe impairment of cognition and confusion, neurological signs), when serum testing for antineuronal antibodies is negative.

Finally, the study was conducted in busy clinical services. Standardised diagnostic instruments were not utilised to obtain the initial diagnosis and there was no use of standardised neuropsychological or functional assessments at follow-up to objectively measure the long-term outcomes.

\section{Clinical significance}

This study suggests a small but significant proportion of people unwell enough to require admission to hospital for their FEP have an illness arising from an autoimmune aetiology and respond to immunotherapy. This study supports recent recommendations for routine antineuronal antibody testing as screening for autoimmune encephalitis in patients admitted with FEP. ${ }^{18,30}$ Obviously, these findings require further replication in other FEP populations and should be combined with rigorous assessment of response to immunotherapy, ideally in the context of randomised placebo-controlled trials.
It is important that research is undertaken with people who are presenting initially with psychosis so as to accurately determine the prevalence of autoimmune neuropsychiatric syndromes in this clinical population. This will guide the nature of clinical investigations that may have particular diagnostic utility in this heterogeneous group.

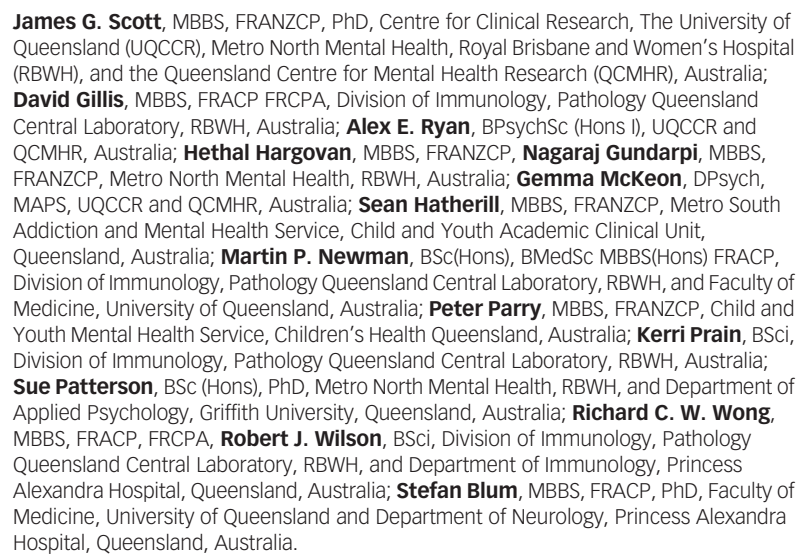
Queensland (UQCCR), Metro North Mental Health, Royal Brisbane and Women's Hospital (RBWH), and the Queensland Centre for Mental Health Research (QCMHR), Australia; David Gillis, MBBS, FRACP FRCPA, Division of Immunology, Pathology Queensland Central Laboratory, RBWH, Australia; Alex E. Ryan, BPsychSC (Hons I), UQCCR and QCMHR, Australia; Hethal Hargovan, MBBS, FRANZCP, Nagaraj Gundarpi, MBBS, QRANZCP, Metro North Mental Health, RBWH, Australi; Gemma McKoon, MANS, UQCCR and MAPS, UQCCR and QCMHR, Australia; Sean Hatherill, MBBS, FRANZCP, Metro South Addiction and Mental Health Service, Child and Youth Academic Clinical Unit, Queensland, Australia; Martin P. Newman, BSC(Hons), BMedSC MBBS(Hons) FRACP, Division of Immunology, Pathology Queensland Central Laboratory, RBWH, and Faculty of Medicine, University of Queensland, Australia; Peter Parry, MBBS, FRANZCP, Child and Youth Mental Health Service, Children's Health Queensland, Australia; Kerri Prain, BSci, Division of Immunology, Pathology Queensland Central Laboratory, RBWH, Australia; Sue Patterson, BSC (Hons), PhD, Metro North Mental Health, RBWH, and Department of Applied Psychology, Griffith University, Queensland, Australia; Richard C. W. Wong, MBBS, FRACP, FRCPA, Robert J. Wilson, BSci, Division of Immunology, Pathology Queensland Central Laboratory, RBWH, and Department of Immunology, Princess Alexandra Hospital, Queensland, Australia; Stefan Blum, MBBS, FRACP, PhD, Faculty of Medicine, University of Queensland and Department of Neurology, Princess Alexandra Hospital, Queensland, Australia.

Correspondence: James G. Scott, MBBS, FRANZCP, PhD, The University of Queensland Centre for Clinical Research, UQ Centre for Clinical Research. Building 71/918 Royal Brisbane and Women's Hospital Campus Herston, Queensland, Australia, 4029. Email: james.scott@health.qld.gov.au

First received 1 Jul 2017, final revision 5 Dec 2017, accepted 21 Jan 2018

Funding

The study was funded by generous support from the Royal Brisbane and Women's Hospital Foundation and from philanthropic donations (BAP Parking and others). JGS is supported by a National Health and Medical Research Council Practitioner Fellowship grant APP1105807.

\section{Acknowledgements}

The authors are grateful to the clinical services and the many clinical staff who supported this study.

\section{References}

1 Farber NB. The NMDA receptor hypofunction model of psychosis. Ann N Y Acad Sci 2003; 1003: 119-30.

2 Dalmau J, Tuzun E, Wu H-y, Masjuan J, Rossi JE, Voloschin A, et al. Paraneoplastic anti-N-methyl-D-aspartate receptor encephalitis associated with ovarian teratoma. Ann Neurol 2007; 61(1): 25-36.

3 Moura M, Silva-Dos-Santos A, Afonso J, Talina M. First-episode psychosis in a 15 year-old female with clinical presentation of anti-NMDA receptor encephalitis: a case report and review of the literature. BMC Res Notes 2016; 9: 374.

4 Kramina S, Kevere L, Bezborodovs N, Purvina S, Rozentals G, Strautmanis J, et al. Acute psychosis due to non-paraneoplastic anti-NMDA-receptor encephalitis in a teenage girl: Case report. Psych J 2015; 4(4): 226-30.

5 Heekin RD, Catalano MC, Frontera AT, Catalano G. Anti-NMDA receptor encephalitis in a patient with previous psychosis and neurological Abnormalities: a diagnostic challenge. Case Rep Psychiatry 2015; 2015: 253891.

6 Lu J, Samson S, Kass J, Ram N. Acute psychosis in a pregnant patient with Graves' hyperthyroidism and anti-NMDA receptor encephalitis. BMJ Case Rep 2015; 2015: bcr2014208052.

7 Tidswell J, Kleinig T, Ash D, Thompson P, Galletly C. Early recognition of anti-Nmethyl D-aspartate (NMDA) receptor encephalitis presenting as acute psychosis. Australas Psychiatry 2013; 21(6): 596-9.

8 Shaaban HS, Choo HF, Sensakovic JW. Anti-NMDA-receptor encephalitis presenting as postpartum psychosis in a young woman, treated with rituximab. Ann Saudi Med 2012; 32(4): 421-3.

9 Mesquita J, Siva L. Anti-NMDA receptor encephalitis suspected as cause of drug-induced psychosis. J Neuropsychiatry Clin Neurosci 2011; 23(4): E2. 
10 Bergink V, Armangue T, Titulaer MJ, Markx S, Dalmau J, Kushner SA Autoimmune encephalitis in postpartum psychosis. Am J Psychiatry 2015 172(9): 901-8.

11 Irani SR, Vincent A. NMDA receptor antibody encephalitis. Curr Neurol Neurosci Rep 2011; 11(3): 298-304.

12 Wandinger K-P, Saschenbrecker S, Stoecker W, Dalmau J. Anti-NMDA-receptor encephalitis: a severe, multistage, treatable disorder presenting with psychosis. J Neuroimmunol 2011; 231(1-2): 86-91.

13 Masdeu JC, Gonzalez-Pinto A, Matute C, Ruiz De Azua S, Palomino A, De Leon J et al. Serum IgG antibodies against the NR1 subunit of the NMDA receptor not detected in schizophrenia. Am J Psychiatry 2012; 169(10): 1120-1.

14 Rhoads J, Guirgis $\mathrm{H}$, McKnight $\mathrm{C}$, Duchemin AM. Lack of anti-NMDA recepto autoantibodies in the serum of subjects with schizophrenia. Schizophr Res 2011; 129(2-3): 213-4.

15 Dalmau J, Lancaster E, Martinez-Hernandez E, Rosenfeld MR, Balice-Gordon R. Clinical experience and laboratory investigations in patients with anti-NMDAR encephalitis. Lancet Neurol 2011; 10(1): 63-74.

16 Steiner J, Walter M, Glanz W, Sarnyai Z, Bernstein HG, Vielhaber S, et al. Increased prevalence of diverse $\mathrm{N}$-methyl-D-aspartate glutamate receptor antbodies in patients with an initial diagnosis of schizophrenia: specific relevance of IgG NR1a antibodies for distinction from N-methyl-D-aspartate glutamate receptor encephalitis. JAMA Psychiatry 2013; 70(3): 271-8.

17 Pruss $\mathrm{H}$, Lennox BR. Emerging psychiatric syndromes associated with antivoltage-gated potassium channel complex antibodies. I Neurol Neurosurg Psychiatry 2016; 87(11): 1242-7.

18 Lennox BR, Palmer-Cooper EC, Pollak T, Hainsworth J, Marks J, Jacobson L, et al. Prevalence and clinical characteristics of serum neuronal cell surface antibodies in first-episode psychosis: a case-control study. Lancet Psychiatry 2017; 4(1): 42-8.

19 Newman MP, Blum S, Wong RC, Scott JG, Prain K, Wilson RJ, et al. Autoimmune encephalitis. Intern Med J 2016; 46(2): 148-57.

20 van Sonderen A, Schreurs MW, de Bruijn MA, Boukhrissi S, Nagtzaam MM Hulsenboom ES, et al. The relevance of VGKC positivity in the absence of LGI1 and Caspr2 antibodies. Neurology 2016; 86(18): 1692-9.

21 van Sonderen A, Petit-Pedrol M, Dalmau J, Titulaer MJ. The value of LGI1, Caspr2 and voltage-gated potassium channel antibodies in encephalitis. Nat Rev Neurol 2017; 13(5): 290-301.
22 Zandi MS, Deakin JB, Morris K, Buckley C, Jacobson L, Scoriels L, et al. Immunotherapy for patients with acute psychosis and serum N-Methyl DAspartate receptor (NMDAR) antibodies: a description of a treated case series. Schizophr Res 2014; 160(1-3): 193-5.

23 Tsutsui K, Kanbayashi T, Tanaka K, Boku S. Anti-NMDA-receptor antibody detected in encephalitis, schizophrenia, and narcolepsy with psychotic features. BMC Psychiatry 2012; 12: 37.

24 Zandi MS, Irani SR, Lang B, Waters $\mathrm{P}$, Jones PB, McKenna $\mathrm{P}$, et al. Diseaserelevant autoantibodies in first episode schizophrenia. J Neurol 2011; 258(4): 686-8.

25 McKeon GL, Scott JG, Spooner DM, Ryan AE, Blum S, Gillis D, et al. Cognitive and social functioning deficits after anti-N-methyl-D-aspartate receptor encephalitis: an exploratory case series. J Int Neuropsychol Soc 2016; 22(8): 828-38.

26 Finke C, Kopp UA, Pruss H, Dalmau J, Wandinger K, Ploner CJ. Cognitive deficits following anti-NMDA receptor encephalitis. J Neurol Neurosurg Psychiatry 2012: 83: 195-8

27 Irani SR, Bera K, Waters P, Zuliani L, Maxwell S, Zandi MS, et al. N-methyl-Daspartate antibody encephalitis: temporal progression of clinical and paraclinical observations in a predominantly non-paraneoplastic disorder of both sexes. Brain 2010; 133(Pt 6): 1655-67.

28 Lang K, Pruss $\mathrm{H}$. Frequencies of neuronal autoantibodies in healthy controls: estimation of disease specificity. Neurol Neuroimmunol Neuroinflammation 2017; 4(5): e386

29 Masdeu JC, Dalmau J, Berman KF. NMDA receptor internalization by autoantibodies: a reversible mechanism underlying psychosis? Trends Neurosci 2016; 39(5): 300-10.

30 Galletly C, Castle D, Dark F, Humberstone V, Jablensky A, Killackey E, et al. Royal Australian and New Zealand College of Psychiatrists clinical practice guidelines for the management of schizophrenia and related disorders. Aust $N \mathrm{ZJ}$ Psychiatry 2016; 50(5): 410-72.

31 World Health Organisation. International Statistical Classification of Diseases and Related Health Problems, 10th Revision (ICD-10). Geneva: WHO 1992. 